\title{
Semiquantitative histologic evaluation improves diagnosis of esophageal carcinoma cuniculatum on biopsy
}

\author{
Derrick Chen ${ }^{1}$, John R Goldblum ${ }^{1}$, Michael Landau ${ }^{1}$, Thomas W Rice ${ }^{2}$, Rish K Pai ${ }^{1}$, \\ Shu-Yuan Xiao ${ }^{3}$ and Xiuli Liu ${ }^{1}$ \\ ${ }^{1}$ Department of Anatomic Pathology, Cleveland Clinic, Cleveland, OH, USA; ${ }^{2}$ Department of Thoracic and \\ Cardiovascular Surgery, Cleveland Clinic, Cleveland, OH, USA and ${ }^{3}$ Department of Pathology, University of \\ Chicago, Chicago, IL, USA
}

\begin{abstract}
Carcinoma cuniculatum, a unique variant of well-differentiated squamous cell carcinoma, has been only rarely reported in the esophagus. The present study was undertaken to determine if a previously observed common histologic pattern for carcinoma cuniculatum is diagnostically useful in esophageal mucosal biopsy specimens. Thirty-five esophageal mucosal biopsies obtained from 25 procedures in 11 patients with a resection-proven diagnosis of carcinoma cuniculatum were compared with 92 esophageal biopsies from 69 patients with benign diagnoses. All biopsies were assessed for the presence of hyperkeratosis, acanthosis, dyskeratosis, deep keratinization, intraepithelial neutrophils, neutrophilic microabscess, focal cytologic atypia, koilocyte-like cells, and keratin-filled cyst/burrows. Each feature, if present, was given one point, and the final histologic score was calculated for each biopsy by summing the points. The mean histologic score was 6.66 (s.d. 1.88) in biopsies from carcinoma cuniculatum vs a mean score of 1.93 (s.d. 1.75) for biopsies with benign diagnoses $(P<0.0001)$. Using a cutoff value of 7 for carcinoma cuniculatum, $57 \%$ of biopsies $(20 / 35)$ from $64 \%$ esophagogastroduodenoscopy procedures $(16 / 25)$ in $91 \%$ patients $(10 / 11)$ would be diagnostic, in comparison to the initial diagnostic rates of carcinoma of 9,12 , and $27 \%$, respectively $(P<0.0001$ for all). None of the 92 benign biopsies showed a score of $\geq 7$. Our results demonstrate that a semiquantitative histologic evaluation of mucosal biopsies taken from an esophageal mass greatly improves the diagnostic sensitivity from patients with carcinoma cuniculatum with $\mathbf{1 0 0} \%$ specificity. Larger studies are necessary to confirm the current findings. Modern Pathology (2013) 26, 806-815; doi:10.1038/modpathol.2012.224; published online 25 January 2013
\end{abstract}

Keywords: candida esophagitis; carcinoma cuniculatum; dyskeratosis; esophagus; hyperkeratosis; mucosal biopsy; neutrophil; squamous cell carcinoma

Carcinoma cuniculatum is a rare variant of squamous cell carcinoma first described in the plantar skin by Ayrd et al in $1954 .{ }^{1}$ Subsequently, it was described in both cutaneous ${ }^{2,3}$ and non-cutaneous sites such as the oral mucosa and larynx ${ }^{4,5}$ Carcinoma cuniculatum has a distinctive morphology characterized by burrowing channels lined by extremely well-differentiated squamous epithelium. ${ }^{3}$

We previously reported nine cases of esophageal carcinoma cuniculatum diagnosed on esophagectomy

Correspondence: Dr X Liu, MD, PhD, Assistant Professor of Pathology, Cleveland Clinic Lerner College of Medicine of Case Western Reserve University, 9500 Euclid Avenue/L25, Cleveland, OH 44195, USA.

E-mail: liux3@ccf.org

Received 22 August 2012; revised 3 December 2012; accepted 5 December 2012; published online 25 January 2013 specimens in seven men and two women during a 20-year period. A common histologic pattern was recognized in this unique variant of welldifferentiated squamous cell carcinoma, including hyperkeratosis, acanthosis, dyskeratosis, deep keratinization, intraepithelial neutrophils, intraepithelial neutrophilic microabscess, focal cytologic atypia, koilocyte-like cells, and keratin-filled cyst/ burrows. ${ }^{6}$ Some of these common features were also observed in two cases of esophageal carcinoma cuniculatum reported by de Petris et al. ${ }^{7}$ However, preoperative diagnosis of this entity is extremely difficult, particularly in small biopsy specimens. This study was undertaken to determine if this common histologic pattern observed in surgically resected esophageal carcinoma cuniculatum is diagnostically useful in esophageal mucosal biopsy specimens. 


\section{Materials and methods}

Our series consisted of nine patients with carcinoma cuniculatum diagnosed on esophagectomy specimens ${ }^{6}$ and two patients with carcinoma cuniculatum diagnosed on endoscopic mucosal resection specimens at the Cleveland Clinic during a 20-year period (1991-2011). All esophageal mucosal biopsies (a total of 35 from 25 esophagogastroduodenoscopic procedures) performed at our institution prior to the definitive diagnosis from these cases were retrieved. In addition, 92 esophageal mucosal biopsies taken from 69 patients were included as controls and had the following clinical conditions: hiatus hernia (5), reflux disease (33), active esophagitis (5), candida esophagitis (2), herpetic esophagitis (1), eosinophilic esophagitis (1), acute graft vs host disease (1), achalasia (4), radiationrelated esophagitis (1), bullous diseases (5), esophageal ulcer (1), non-specific small $(<1 \mathrm{~cm}$ nodule) nodule or nodules (4), benign-appearing, intrinsic stricture (2), non-obstructive Schatzki ring (2), and normal (2). The histologic interpretations of these biopsies were as follows: normal esophagus (28), reflux-induced reactive changes (30), reflux esophagitis (10), active esophagitis of unknown etiology (11), esophageal ulcer (3), candida esophagitis (4), herpetic esophagitis (1), eosinophilic esophagitis (2), and others (3). Among the control group, nine biopsies were from five patients with bullous diseases (not further specified) diagnosed between 1997 and 2008; the remaining 83 specimens were consecutive esophageal biopsies from 64 adult patients encountered during a 1-month period in 2011.

The study was approved by the Institutional Review Board of Cleveland Clinic.

All surgical pathology reports and esophageal biopsy slides were retrieved and reviewed by a gastrointestinal pathologist (XL). All cases were evaluated for the following features: hyperkeratosis (either parakeratosis and/or orthokeratosis), acanthosis (defined as overall squamous epithelial hyperplasia), dyskeratosis (defined as the presence of individual apoptotic keratinocytes), deep keratinization, intraepithelial neutrophils (defined as the presence of neutrophils in the neoplastic epithelium), intraepithelial neutrophilic microabscess (defined as the presence of six or more neutrophils with associated epithelial injury), cytologic atypia, koilocyte-like cells, and keratin-filled cyst/burrows. Each feature, if present, was given 1 point, and the final histologic score was calculated for each biopsy by summing up the points.

In addition, the presence of intraepithelial lymphocytosis (defined as the presence of $\geq 15$ lymphocytes per high power field in the neoplastic epithelium), was also assessed. Further, the presence of Candida and bacterial overgrowth was also assessed on hematoxylin and eosin stain and/or Grocott's methenamine silver stain.

Clinical features obtained by chart review included the patients' age, gender, type and duration of symptoms, tumor location, and endoscopic appearance. Endoscopic ultrasound results were also obtained when available. In the control group, esophagogastroduodenoscopic reports were available for 72 procedures (of 74, 97\%) and none had mucosal mass lesions.

\section{Statistical Analysis}

In statistical analysis, $\chi^{2}$ test was performed for categorical data and the frequency of each feature. Student $t$-test was used to analyze continuous data. The results for patient age were expressed as mean \pm s.d. All $P$-values were two-sided, and statistical significance was set at $P \leq 0.05$.

\section{Results}

\section{Demographics and Clinical Features}

As shown in Table 1, our series consisted of 11 patients including nine previously reported cases $^{6}$ and two additional cases (cases 10 and 11). Seven patients were males and four were females. The age of the patients ranged from 40 to 72 years (mean 57 years; s.d. 12 years). Eleven tumors from nine patients were diagnosed on esophagectomy specimens and two tumors from two patients on endoscopic mucosal resection specimens (cases 10 and 11). Of the 11 patients, $10(91 \%)$ had dysphagia, 7 (64\%) had a smoking history, $3(27 \%)$ had a history of excessive ethanol use, and $8(73 \%)$ had either gastroesophageal reflux disease or prior treatment with anti-reflux medication. For each of the 11 patients, an esophagogastroduodenoscopic report documented an esophageal mass. The location of the mass was in the cervical/mid-esophagus for two patients, in the mid-esophagus for two patients, in the distal esophagus for five patients, and in the distal esophagus/gastroesophageal junction for two patients. Endoscopic ultrasound examination was performed for eight patients and showed evidence of wall invasion in all patients and findings suspicious for nodal metastasis in four patients. Of the nine patients who underwent esophagectomy, two patients died post-operatively due to complications; the remaining patients were followed up for a median duration of 84 months (48-214 months). During the follow-up period, three patients died at 49, 66, and 214 months after esophagectomy at the ages of 66, 68, and 91 years, respectively, from causes unrelated to recurrent or metastatic esophageal cancer. Four patients were alive without disease at 48, 49, 84, and 87 months post-esophagectomy. Of the two patients with endoscopic mucosal resection-proven carcinoma cuniculatum treated with radiation, one was alive at 19 months without disease and the other patient (case 10) had tracheal recurrence at 6 months after the diagnosis and died 2 months later. 
Table 1 Demographics and clinical and endoscopic features of resection-proven esophageal carcinoma cuniculatum

\begin{tabular}{|c|c|c|c|c|c|c|c|c|c|c|c|c|c|c|c|c|}
\hline Case & $\begin{array}{c}\text { Age at } \\
\text { diagnosis }\end{array}$ & Sex & $\begin{array}{l}\text { Esophageal } \\
\text { symptoms }\end{array}$ & $\begin{array}{l}\text { Other } \\
\text { symptoms }\end{array}$ & $\begin{array}{l}\text { Smoking } \\
\text { history }\end{array}$ & $\begin{array}{l}\text { Ethanol } \\
\text { history }\end{array}$ & $\begin{array}{l}\text { Reflux } \\
\text { history }\end{array}$ & Location & $\begin{array}{l}\text { Appearance of } \\
\text { lesion }\end{array}$ & $\begin{array}{l}\text { Wall } \\
\text { invasion }\end{array}$ & $\begin{array}{l}\text { Endoscopic } \\
\text { diagnosis }\end{array}$ & $\begin{array}{l}\text { Clinical } \\
\text { diagnosis }\end{array}$ & $\begin{array}{l}\text { Follow- } \\
\text { up }\end{array}$ & $\begin{array}{c}\text { Age } \\
\text { at } \\
\text { death }\end{array}$ & $\begin{array}{l}\text { Survival } \\
\text { after } \\
\text { diagnosis }\end{array}$ & $\begin{array}{l}\text { Cause of } \\
\text { death }\end{array}$ \\
\hline 1 & 63 & M & $\begin{array}{l}\text { Dysphagia } \\
\text { (6 months) }\end{array}$ & $\begin{array}{l}\text { Diarrhea } \\
\text { Weight loss }\end{array}$ & $\begin{array}{l}72 \text { pack- } \\
\text { year }\end{array}$ & - & - & Distal & $\begin{array}{l}\text { Verrucous mass } \\
\text { causing severe } \\
\text { stricture }\end{array}$ & Yes & $\begin{array}{l}\text { Esophageal } \\
\text { Crohn's disease }\end{array}$ & $\begin{array}{l}\text { Esophageal } \\
\text { Crohn's } \\
\text { disease }\end{array}$ & Dead & 63 & 1 day & $\begin{array}{l}\text { Ventricular } \\
\text { fibrillation }\end{array}$ \\
\hline 2 & 40 & M & $\begin{array}{l}\text { Dysphagia } \\
\text { (long } \\
\text { duration) }\end{array}$ & Weight loss & $\begin{array}{l}8.5 \text { pack- } \\
\text { year }\end{array}$ & - & * & Distal & Frond-like mass & $\begin{array}{l}\text { Yes } \\
\text { (thickening } \\
\text { of mucosa } \\
\text { and } \\
\text { submucosa) }\end{array}$ & ? Carcinoma & $\begin{array}{l}\text { Achalasia, } \\
\text { malignant } \\
\text { neoplasm of } \\
\text { esophagus? }\end{array}$ & Dead & 40 & 19 days & $\begin{array}{l}\text { Acute } \\
\text { respiratory } \\
\text { distress } \\
\text { syndrome }\end{array}$ \\
\hline 3 & 73 & M & - & $\begin{array}{l}\text { Change in } \\
\text { bowel habits } \\
\text { (3 months) }\end{array}$ & - & - & NA & $\begin{array}{l}\text { Distal/ } \\
\text { Gastroesophageal } \\
\text { junction }\end{array}$ & $\begin{array}{l}\text { Mass, not further } \\
\text { detailed }\end{array}$ & $\mathrm{NA}$ & NA & $\begin{array}{l}\text { Carcinoma of } \\
\text { the stomach }\end{array}$ & Dead & 91 & $\begin{array}{l}214 \\
\text { months }\end{array}$ & NA \\
\hline 4 & 46 & M & $\begin{array}{l}\text { Dysphagia } \\
\text { (3 months) }\end{array}$ & $\begin{array}{l}\text { Weight loss, } \\
\text { mid-chest } \\
\text { discomfort }\end{array}$ & $\begin{array}{l}50 \text { pack- } \\
\text { year }\end{array}$ & + & * & Distal & $\begin{array}{l}\text { Fungating } \\
\text { ulcerated mass }\end{array}$ & Yes & $\begin{array}{l}\text { Esophageal } \\
\text { carcinoma }\end{array}$ & $\begin{array}{l}\text { Esophageal } \\
\text { carcinoma }\end{array}$ & Alive & N/A & $\begin{array}{l}157 \\
\text { months }\end{array}$ & N/A \\
\hline 5 & 62 & M & $\begin{array}{l}\text { Dysphagia } \\
\text { (5 years) }\end{array}$ & $\begin{array}{l}\text { Epigastric } \\
\text { pain, } \\
\text { Regurgitation }\end{array}$ & $\begin{array}{l}45 \text { pack- } \\
\text { year }\end{array}$ & - & + & $\begin{array}{l}\text { Distal/ } \\
\text { Gastroesophageal } \\
\text { junction }\end{array}$ & $\begin{array}{l}\text { Irregular } \\
\text { ulcerated mass }\end{array}$ & Yes & $\begin{array}{l}\text { Stenotic } \\
\text { submucosal } \\
\text { lesion }\end{array}$ & $\begin{array}{l}\text { Esophageal } \\
\text { cancer? }\end{array}$ & Dead & 66 & $\begin{array}{l}49 \\
\text { months }\end{array}$ & $\begin{array}{l}\text { Pleural } \\
\text { empyema } \\
\text { with } \\
\text { respiratory } \\
\text { failure }\end{array}$ \\
\hline 6 & 44 & M & $\begin{array}{l}\text { Dysphagia } \\
\text { (5 months) }\end{array}$ & $\begin{array}{l}\text { Weight loss, } \\
\text { regurgitation }\end{array}$ & $\begin{array}{l}30 \text { pack- } \\
\text { year }\end{array}$ & - & * & Distal & $\begin{array}{l}\text { Large, fungating, } \\
\text { friable, irregular } \\
\text { mass }\end{array}$ & Yes & Cancer & $\begin{array}{l}\text { Squamous } \\
\text { cell } \\
\text { carcinoma of } \\
\text { the } \\
\text { esophagus? }\end{array}$ & Alive & N/A & $\begin{array}{l}87 \\
\text { months }\end{array}$ & N/A \\
\hline 7 & 48 & $\mathrm{~F}$ & $\begin{array}{l}\text { Dysphagia } \\
\text { (since } \\
\text { childhood) }\end{array}$ & $\begin{array}{l}\text { Weight loss } \\
\text { (after } \\
\text { perforation } \\
\text { repair) }\end{array}$ & $\begin{array}{l}3 \text { pack- } \\
\text { year }\end{array}$ & + & * & $25 \mathrm{~cm}(\mathrm{Mid})^{\mathrm{a}}$ & $\begin{array}{l}\text { Polypoid, } \\
\text { strictured, lesion } \\
\text { with whitish } \\
\text { ulcerated area }\end{array}$ & ND & ? Malignancy & $\begin{array}{l}\text { Esophageal } \\
\text { stricture }\end{array}$ & Alive & N/A & $\begin{array}{l}84 \\
\text { months }\end{array}$ & N/A \\
\hline 8 & 63 & M & $\begin{array}{l}\text { Dysphagia } \\
\text { (unknown } \\
\text { duration) }\end{array}$ & - & $\begin{array}{l}40 \text { pack- } \\
\text { year }\end{array}$ & + & + & Distal & $\begin{array}{l}\text { Polypoid } \\
\text { obstructing } \\
\text { lesion }\end{array}$ & Yes & $\begin{array}{l}\text { Circumferential } \\
\text { stricture }\end{array}$ & $\begin{array}{l}\text { Esophageal } \\
\text { carcinoma? }\end{array}$ & Dead & 68 & $\begin{array}{l}66 \\
\text { months }\end{array}$ & $\begin{array}{l}\text { Lung cancer } \\
\text { metastasis to } \\
\text { brain }\end{array}$ \\
\hline 9 & 72 & $\mathrm{~F}$ & $\begin{array}{l}\text { Dysphagia } \\
\text { (6 months) }\end{array}$ & Odynophagia & - & - & - & $25-27 \mathrm{~cm}$ & $\begin{array}{l}\text { Several warty } \\
\text { lesions }\end{array}$ & Yes & $\begin{array}{l}\text { Esophageal } \\
\text { masses }\end{array}$ & $\begin{array}{l}\text { Squamous } \\
\text { cell } \\
\text { carcinoma of } \\
\text { esophagus }\end{array}$ & Alive & N/A & $\begin{array}{l}48 \\
\text { months }\end{array}$ & N/A \\
\hline 10 & 67 & $\mathrm{~F}$ & $\begin{array}{l}\text { Dysphagia } \\
\text { (long } \\
\text { duration) }\end{array}$ & - & - & - & * & $12-30 \mathrm{~cm}$ & $\begin{array}{l}\text { Obstructing } \\
\text { mass }\end{array}$ & ND & $\begin{array}{l}\text { Esophageal } \\
\text { mass }\end{array}$ & $\begin{array}{l}\text { Verrucous } \\
\text { carcinoma? }\end{array}$ & Dead & 48 & 8 months & $\begin{array}{l}\text { Tracheal } \\
\text { recurrence }\end{array}$ \\
\hline 11 & 48 & $\mathrm{~F}$ & $\begin{array}{l}\text { Dysphagia } \\
\text { (5 years) }\end{array}$ & Weight loss & - & - & * & $15-28 \mathrm{~cm}$ & Nodular mass & $\begin{array}{l}\text { Yes } \\
\text { (thickening } \\
\text { of all layers) }\end{array}$ & $\begin{array}{l}\text { Verrucous } \\
\text { neoplasm vs } \\
\text { esophagitis with } \\
\text { stricture }\end{array}$ & $\begin{array}{l}\text { Esophageal } \\
\text { cancer? }\end{array}$ & Alive & N/A & $\begin{array}{l}19 \\
\text { months }\end{array}$ & N/A \\
\hline
\end{tabular}

Note: +: present; - : absent; *: patient has been on proton pump inhibitor.

NA: no detailed information available.

N/A: not applicable.

ND: not done or not mentioned in endoscopic report.

a The epicenter of the tumor was in the distal esophagus. The tumor extended proximally and partially involved the mid esophagus. 
A total of 35 esophageal mucosal biopsies from 25 endoscopic procedures were performed at our institution for 11 patients prior to the definitive diagnosis by either esophagectomy or endoscopic mucosal resection. The number of mucosal biopsies performed ranged from 1 to 8 with a mean of three biopsies per patient prior to the definite diagnosis. As shown in Table 2, all but three patients had multiple endoscopic procedures prior to the definite diagnosis. The original diagnoses rendered on the endoscopic mucosal biopsy are shown in Table 2. Despite the clinical impression of esophageal malignancy, a definite diagnosis of esophageal squamous cell carcinoma was achieved in only 3 of $11(27 \%)$ patients (cases 1, 2, and 7), from three procedures (of $25,12 \%$ ) and three biopsies (of $35,9 \%$ ), prior to esophagectomy or endoscopic mucosal resection. An atypical squamous proliferation was diagnosed in six biopsies from an additional three patients and in one biopsy from one patient who had a definitive diagnosis rendered in a separate biopsy taken during the same endoscopic procedure (case 7). Of note, one case (case 4) showed persistent squamous atypia in all three biopsies from three procedures.

The control group consisted of 92 esophageal mucosal biopsies with benign diagnoses from 69 patients including 39 males and 30 females with an age range from 18 to 89 years (mean 59 years (s.d. 16 years)). The age and gender composition were not significantly different between the carcinoma cuniculatum and control groups $(P=0.65$ and 0.75 , respectively).

\section{Histomorphologic Features of Carcinoma Cuniculatum of the Esophagus in Resected Specimens}

Histologically reviewing the 13 tumors of resectionproven carcinoma cuniculatum revealed a common histologic pattern, as we reported previously ${ }^{6}$ (Table 3; Figure 1a and b). Specifically, the welldifferentiated squamous epithelium demonstrated hyperkeratosis (either parakeratosis or orthokeratosis) in 13 tumors (of 13, 100\%), acanthosis in 13 tumors (of 13,100\%), dyskeratosis in 13 tumors (of $13,100 \%$ ), deep keratinization in 13 tumors (of 13, $100 \%)$, intraepithelial neutrophils in 13 tumors (of 13,100\%), intraepithelial neutrophilic microabscess in 12 tumors (of 13, 92\%), atypia in 13 tumors (of 13,100\%), keratin cyst/burrow in 13 tumors (of 13, 100\%), and koilocyte-like cells in 13 tumors (of 13, 100\%). None of the tumors had abnormal mitoses. Candida, bacterial overgrowth, granular cell layer, and intraepithelial lymphocytosis were seen in 15, 54, 15, and 15\% tumors, respectively. The resection-proven cases of esophageal carcinoma cuniculatum had a mean histologic score of 8.91 (s.d. 0.3, calculated by summing all points for hyperkeratosis, acanthosis, dyskeratosis, deep keratinization, intraepithelial neutrophils, intraepithelial neutrophilic microabs- cess, focal cytologic atypia, and koilocyte-like cell as described in the Materials and methods).

\section{Histomorphologic Features of Carcinoma Cuniculatum in Esophageal Mucosal Biopsy in Comparison to those in Esophagectomy Specimens}

A total of 35 esophageal mucosal biopsies from 25 esophagogastroduodenoscopy procedures were reviewed and assessed as described in Materials and methods. As demonstrated in Table 3 and Figure 1a, hyperkeratosis, acanthosis, dyskeratosis, intraepithelial neutrophils, intraepithelial neutrophilic microabscess, and koilocyte-like cells, were seen in $97,100,91,86,74$, and $80 \%$ of preoperative esophageal biopsies from patients with carcinoma cuniculatum, respectively. These frequencies were not significantly different from the frequencies of 100 , $100,100,100,92$, and $100 \%$ for each feature in resection-proven tumors $(P=1.0,1.0,0.55,0.30$, 0.24 , and 0.166 , respectively). However, the endoscopic mucosal biopsies were less likely to show deep keratinization, cytologic atypia, and keratin cyst/burrow than resection specimens (63 vs 100\%, $P=0.01$; 51 vs $100 \%, P=0.0015$; and 23 vs $100 \%$, $P=0.000001$ ) (Table 3; Figure 1b). Common histologic features in endoscopic mucosal biopsies of esophageal carcinoma cuniculatum are shown in Figure 2a-d. None of the cases showed abnormal mitoses. The mean histologic score on mucosal biopsies from patients with carcinoma cuniculatum was 6.66 (s.d. 1.88), significantly lower than that in resection specimens $(8.91 \pm 0.3, P=0.0003)$.

\section{Histomorphologic Features of Carcinoma Cuniculatum in Esophageal Mucosal Biopsy in Comparison to Esophageal Mucosal Biopsies with Benign Diseases}

The frequencies of hyperkeratosis, acanthosis, dyskeratosis, deep keratinization, intraepithelial neutrophils, intraepithelial neutrophilic microabscess, cytological atypia, and koilocyte-like cells in endoscopic mucosal biopsy from patients with resectionproven esophageal carcinoma cuniculatum were higher than those seen in the control group (97 vs $48 \%, 100$ vs $43 \%$, 91 vs $12 \%$, 63 vs $1 \%$, 86 vs $25 \%$, 74 vs $9 \%$, 51 vs $10 \%$, 80 vs $41 \%$, respectively; $P<0.05$ for all features) (Figure 1a and b). Keratinfilled cysts/burrows were present in $23 \%$ biopsies with carcinoma cuniculatum and $3 \%$ biopsies with benign diagnoses $(P=0.001)$.

Biopsies were also reviewed for the presence of fungal organisms, bacteria, presence of a granular cell layer, and intraepithelial lymphocytes. Candida organisms were seen in 9 of $35(26 \%)$ biopsies from 7 patients (of 11,64\%) and bacterial overgrowth was observed in 16 of 35 (46\%) biopsies from 7 patients (of 11, 64\%). A granular cell layer was focally present in $4(11 \%)$ biopsies from four carcinoma 
Table 2 Pathologic findings on endoscopic mucosal biopsies prior to esophagectomy or EMR in patients with esophageal carcinoma cuniculatum

\begin{tabular}{|c|c|c|c|c|c|c|c|c|c|c|c|c|c|}
\hline Case & $\begin{array}{l}\text { Date of } \\
\text { procedure }\end{array}$ & Parts & $\begin{array}{c}\text { Hyperkeratosis } \\
\text { (presence=1; } \\
\text { absence }=0)\end{array}$ & $\begin{array}{c}\text { Acanthosis } \\
\text { (presence=1; } \\
\text { absence =0) }\end{array}$ & $\begin{array}{l}\text { Dyskeratosis } \\
\text { (presence=1; } \\
\text { absence }=0)\end{array}$ & $\begin{array}{l}\text { Deep } \\
\text { keratinization } \\
\text { (presence=1; } \\
\text { absence }=0 \text { ) }\end{array}$ & $\begin{array}{l}\text { Intraepithelial } \\
\text { neutrophils } \\
\text { (presence=1; } \\
\text { absence=0) }\end{array}$ & $\begin{array}{l}\text { Neutrophilic } \\
\text { microabscess } \\
\text { (presence=1; } \\
\text { absence=0) }\end{array}$ & $\begin{array}{c}\text { Atypia } \\
\text { (presence=1; } \\
\text { absence }=0)\end{array}$ & $\begin{array}{l}\text { Keratin cyst/ } \\
\text { burrow } \\
\text { (presence }=1 ; \\
\text { absence }=0 \text { ) }\end{array}$ & $\begin{array}{c}\text { Koilocyte-like } \\
\text { cells } \\
\text { (presence=1; } \\
\text { absence }=0 \text { ) }\end{array}$ & $\begin{array}{l}\text { Histologic } \\
\text { score }\end{array}$ & Original diagnosis \\
\hline$\# 1-1$ & 5/13/1993 & & 1 & 1 & 1 & 1 & 1 & 1 & 1 & 0 & 1 & 8 & \multirow{5}{*}{$\begin{array}{l}\text { Invasive, moderately-differentiated } \\
\text { squamous cell carcinoma } \\
\text { Invasive, well-differentiated, } \\
\text { squamous cell carcinoma } \\
\text { Mildly inflamed squamous mucosa } \\
\text { Non-specific inflammation }\end{array}$} \\
\hline \#2-1 & 3/6/1998 & & 1 & 1 & 1 & 1 & 1 & 1 & 1 & 0 & 1 & 8 & \\
\hline \#3-3 & $11 / 3 / 2003$ & & 1 & 1 & 1 & 0 & 1 & 1 & 0 & 0 & 1 & 6 & \\
\hline \multirow[t]{2}{*}{ \#3-2 } & $10 / 2 / 2003$ & Part & 1 & 1 & 1 & 1 & 1 & 1 & 1 & 0 & 1 & 8 & \\
\hline & & $\begin{array}{l}\text { Part } \\
\text { B }\end{array}$ & 1 & 1 & 1 & 1 & 1 & 0 & 1 & 0 & 1 & 7 & \\
\hline \#3-1 & $8 / 27 / 2003$ & & 1 & 1 & 1 & 0 & 1 & 0 & 1 & 0 & 0 & 5 & \multirow{11}{*}{$\begin{array}{l}\text { Active esophagitis, c/w candidiasis } \\
\text { Superficial fragment of squamous } \\
\text { epithelium with cytologic atypia } \\
\text { Atypical squamous epithelium, } \\
\text { ulcer, candidas species } \\
\text { Ulcer with acute inflammation and } \\
\text { necrosis, detached atypical } \\
\text { squamous epithelium } \\
\text { Active esophagitis } \\
\text { Active esophagitis with ulcer; } \\
\text { reactive change; candida species } \\
\text { Parakeratosis and atypical } \\
\text { paradoxical maturation change } \\
\text { Fungal esophagitis } \\
\text { Atypical papillary squamous } \\
\text { hyperplasiais suspicious but not } \\
\text { diagnostic of squamous cell } \\
\text { carcinoma } \\
\text { Invasive, well-differentiated, } \\
\text { squamous cell carcinoma }\end{array}$} \\
\hline \#4-3 & $1 / 15 / 2004$ & & & & & 1 & 1 & 0 & & & & & \\
\hline \#4-2 & $1 / 2 / 2004$ & & 1 & 1 & 1 & 1 & 1 & 1 & 0 & 0 & 1 & 7 & \\
\hline \#4-1 & $\begin{array}{l}12 / 19 / \\
2003\end{array}$ & & 1 & 1 & 1 & 1 & 1 & 1 & 1 & 0 & 1 & 8 & \\
\hline \#5-1 & $3 / 25 / 2004$ & & 1 & 1 & 1 & 0 & 1 & 0 & 0 & 0 & 0 & 4 & \\
\hline \#6-3 & $5 / 12 / 2005$ & & 1 & 1 & 1 & 0 & 1 & 1 & 0 & 0 & 1 & 6 & \\
\hline \#6-2 & $4 / 26 / 2005$ & & 1 & 1 & 1 & 1 & 0 & 1 & 0 & 0 & 1 & 6 & \\
\hline$\# 6-1$ & 2/28/2005 & & 1 & 1 & 1 & 1 & 1 & 1 & 0 & 0 & 1 & 7 & \\
\hline \multirow[t]{2}{*}{$\# 7-2$} & $3 / 22 / 2007$ & $\begin{array}{l}\text { Part } \\
\text { A }\end{array}$ & 1 & 1 & 1 & 1 & 1 & 1 & 1 & 1 & 1 & 9 & \\
\hline & & $\begin{array}{l}\text { Part } \\
\text { B }\end{array}$ & 1 & 1 & 1 & 1 & 1 & 1 & 1 & 1 & 1 & 9 & \\
\hline \multirow[t]{3}{*}{ \#7-1 } & & Part & 0 & 1 & 0 & 0 & 0 & 0 & 0 & 0 & 0 & 1 & \\
\hline & $3 / 8 / 2007$ & $\begin{array}{l}\text { Part } \\
\text { C }\end{array}$ & 1 & 1 & 1 & 1 & 1 & 1 & 0 & 1 & 1 & 8 & \multirow[t]{2}{*}{$\begin{array}{l}\text { Atypical papillary (verrucous) } \\
\text { squamous hyperplasia, } \\
\text { hyperkeratosis, parakeratosis, } \\
\text { actively inflamed (indefinite for } \\
\text { dysplasia) }\end{array}$} \\
\hline & & Part & 1 & 1 & 0 & 0 & 1 & 0 & 0 & 0 & 0 & 3 & \\
\hline \#8-2 & $2 / 17 / 2010$ & & 1 & 1 & 1 & 0 & 0 & 0 & 0 & 1 & 1 & 5 & Squamo-proliferation, favor \\
\hline$\# 8-1$ & $1 / 29 / 2010$ & & 1 & 1 & 1 & 1 & 1 & 1 & 1 & 0 & 1 & 8 & $\begin{array}{l}\text { Treactive } \\
\text { Fungal esophagitis }\end{array}$ \\
\hline \multirow[t]{5}{*}{ \#9-4 } & $\begin{array}{l}12 / 28 / \\
2009\end{array}$ & $\begin{array}{l}\text { Part } \\
\text { A }\end{array}$ & 1 & 1 & 1 & 0 & 1 & 1 & 0 & 0 & 1 & 6 & $\begin{array}{l}\text { Ulceration with associated } \\
\text { necrosis, acute inflammation, } \\
\text { bacterial overgrowth, and } \\
\text { regenerative change }\end{array}$ \\
\hline & & Part & 1 & 1 & 1 & 0 & 1 & 1 & 1 & 0 & 0 & 6 & \\
\hline & & Part & 1 & 1 & 1 & 1 & 1 & 1 & 1 & 0 & 1 & 8 & \\
\hline & & $\begin{array}{l}\text { Cart } \\
\text { Part }\end{array}$ & 1 & 1 & 1 & 1 & 1 & 1 & 1 & 1 & 1 & 9 & \\
\hline & & $\begin{array}{l}\text { Dart } \\
\text { Part }\end{array}$ & 1 & 1 & 1 & 1 & 1 & 1 & 1 & 0 & 1 & 8 & \\
\hline$\# 9-3$ & $10 / 7 / 2009$ & & 1 & 1 & 1 & 1 & 1 & 1 & 0 & 1 & 1 & 8 & \multirow{2}{*}{$\begin{array}{l}\text { Ulcerated active esophagitis with } \\
\text { reactive squamous hyperplasia } \\
\text { Active esophagitis with squamous } \\
\text { hyperplasia; fungal organisms } \\
\text { present }\end{array}$} \\
\hline$\# 9-2$ & $1 / 2 / 2008$ & & 1 & 1 & 1 & 1 & 1 & 1 & 1 & 1 & 0 & 8 & \\
\hline
\end{tabular}


Table 2 (Continued)

\begin{tabular}{|c|c|c|c|c|c|c|c|c|c|c|c|c|c|}
\hline Case & $\begin{array}{l}\text { Date of } \\
\text { procedure }\end{array}$ & Parts & $\begin{array}{c}\text { Hyperkeratosis } \\
\text { (presence=1; } \\
\text { absence =0) }\end{array}$ & $\begin{array}{c}\text { Acanthosis } \\
\text { (presence=1; } \\
\text { absence =0) }\end{array}$ & $\begin{array}{c}\text { Dyskeratosis } \\
(\text { presence=1; } \\
\text { absence }=0)\end{array}$ & $\begin{array}{c}\text { Deep } \\
\text { keratinization } \\
\text { (presence=1; } \\
\text { absence }=0 \text { ) }\end{array}$ & $\begin{array}{c}\text { Intraepithelial } \\
\text { neutrophils } \\
\text { (presence=1; } \\
\text { absence }=0 \text { ) }\end{array}$ & $\begin{array}{c}\text { Neutrophilic } \\
\text { microabscess } \\
\text { (presence=1; } \\
\text { absence =0) }\end{array}$ & $\begin{array}{c}\text { Atypia } \\
\text { (presence =1; } \\
\text { absence }=0 \text { ) }\end{array}$ & $\begin{array}{c}\text { Keratin cyst/ } \\
\text { burrow } \\
\text { (presence }=1 ; \\
\text { absence }=0 \text { ) }\end{array}$ & $\begin{array}{c}\text { Koilocyte-like } \\
\text { cells } \\
\text { (presence }=1 ; \\
\text { absence }=0 \text { ) }\end{array}$ & $\begin{array}{l}\text { Histologic } \\
\text { score }\end{array}$ & Original diagnosis \\
\hline$\# 9-1$ & $7 / 21 / 2005$ & & 1 & 1 & 1 & 0 & 1 & 1 & 0 & 0 & 1 & 6 & $\begin{array}{l}\text { Active esophagitis with ulcer and } \\
\text { numerous candida species }\end{array}$ \\
\hline \multirow[t]{2}{*}{ \#10-3 } & $6 / 22 / 2010$ & $\begin{array}{l}\text { Part } \\
\text { A }\end{array}$ & 1 & 1 & 1 & 0 & 1 & 1 & 0 & 0 & 1 & 6 & $\begin{array}{l}\text { Active esophagitis with reactive } \\
\text { epithelial changes; rare fungal } \\
\text { hyphae }\end{array}$ \\
\hline & & $\begin{array}{l}\text { Part } \\
\text { B }\end{array}$ & 1 & 1 & 1 & 1 & 1 & 1 & 1 & 0 & 1 & 8 & \\
\hline$\# 10-2$ & 5/19/2010 & & 1 & 1 & 1 & 1 & 0 & 1 & 0 & 0 & 0 & 5 & $\begin{array}{l}\text { Hyperplastic squamous mucosa } \\
\text { with acute and chronic } \\
\text { inflammation }\end{array}$ \\
\hline \multirow[t]{2}{*}{$\# 10-1$} & $5 / 12 / 2000$ & $\begin{array}{l}\text { Part } \\
\text { A }\end{array}$ & 1 & 1 & 0 & 0 & 0 & 0 & 0 & 0 & 1 & 3 & Mild acute inflammation \\
\hline & & $\begin{array}{l}\text { Part } \\
\text { B }\end{array}$ & 1 & 1 & 1 & 0 & 1 & 1 & 1 & 0 & 1 & 7 & $\begin{array}{l}\text { Ulcerated squamous mucosa; rare } \\
\text { budding yeast }\end{array}$ \\
\hline \#11-2 & $12 / 9 / 2010$ & & 1 & 1 & 1 & 1 & 1 & 1 & 1 & 1 & 1 & 9 & $\begin{array}{l}\text { Acute esophagitis with superficial } \\
\text { erosion or ulcer; squamous } \\
\text { hyperplasia; reactive changes }\end{array}$ \\
\hline \multirow[t]{2}{*}{$\# 11-1$} & $11 / 5 / 2010$ & & 1 & 1 & 1 & 1 & 1 & 0 & 1 & 0 & 1 & 7 & $\begin{array}{l}\text { Markedly inflamed atypical } \\
\text { squamo-proliferative lesion }\end{array}$ \\
\hline & & $\begin{array}{l}\text { Total } \\
\text { (35) }\end{array}$ & 34 (97\%) & $35(100 \%)$ & $32(91 \%)$ & $22(63 \%)$ & $30(86 \%)$ & $26(74 . \%)$ & $18(51 \%)$ & $8(23 \%)$ & $28(80 \%)$ & $8.91 \pm 0.30$ & \\
\hline
\end{tabular}

Table 3 Histomorphologic features of esophageal carcinoma cuniculatum on esophagectomy or EMR specimens

\begin{tabular}{|c|c|c|c|c|c|c|c|c|c|c|c|c|c|c|c|}
\hline Case & Specimen & $\begin{array}{c}\text { Hyper- } \\
\text { keratosis }\end{array}$ & Acanthosis & Dyskeratosis & $\begin{array}{c}\text { Deep } \\
\text { keratinization }\end{array}$ & IEN & $N M$ & Atypia $^{\mathrm{a}}$ & $\begin{array}{c}\text { Keratin cyst/ } \\
\text { burrow }\end{array}$ & $\begin{array}{l}\text { Koilocyte- } \\
\text { like cells }\end{array}$ & $\begin{array}{c}\text { Abnormal } \\
\text { mitoses }\end{array}$ & Candida & $\begin{array}{c}\text { Bacterial } \\
\text { overgrowth }\end{array}$ & $\begin{array}{l}\text { Granular cell } \\
\text { layer }\end{array}$ & IEL \\
\hline 1 & Esophagectomy & + & + & + & + & $+1-$ & - & + & + & + & - & + (rare) & + & - & - \\
\hline 2 & Esophagectomy & + & + & + & + & + & + & + & + & + & - & - & + & - & $+1-$ \\
\hline 3 & Esophagectomy & + & + & + & + & + & + & Mild & + & + & - & - & - & + & - \\
\hline 4 & Esophagectomy & + & + & + & + & $+1-$ & $+1-$ & Focal & + & + & - & - & - & - & - \\
\hline 5 & Esophagectomy & + & + & + & + & + & + & Mild & + & + & - & - & $+1-$ & + & - \\
\hline 6 & Esophagectomy & + & + & + & + & + & + & + & + & + & - & + & + & - & - \\
\hline 7 & Esophagectomy & + & + & + & + & + & + & + & + & + & - & - & + & - & $+1-$ \\
\hline 8 & Esophagectomy & + & + & + & + & $+1-$ & $+1-$ & + & + & + & - & - & + & - & - \\
\hline \multirow[t]{3}{*}{9} & $\begin{array}{l}\text { Esophagectomy } \\
\text { Tumor \#1 }\end{array}$ & $+1-$ & + & + & + & + & + & Mild & $+1-$ & + & - & - & + & - & - \\
\hline & Tumor \#2 & + & + & $+1-$ & + & + & + & Mild & $+1-$ & + & - & - & - & - & - \\
\hline & Tumor \#3 & + & + & $+1-$ & + & + & + & Mild & $+1-$ & + & - & - & - & - & - \\
\hline 10 & EMR & + & + & + & + & + & + & + & + & + & - & - & - & - & - \\
\hline 11 & EMR & + & + & + & + & + & + & + & + & + & - & - & - & - & - \\
\hline $\begin{array}{l}\text { Total (13 } \\
\text { tumors) }\end{array}$ & & $100 \%$ & $100 \%$ & $100 \%$ & $100 \%$ & $100 \%$ & $92 \%$ & $100 \%$ & $100 \%$ & $100 \%$ & $100 \%$ & $15 \%$ & $54 \%$ & $15 \%$ & $15 \%$ \\
\hline
\end{tabular}

IEN: intraepithelial neutrophil; NM: intraepithelial neutrophilic microabscess; IEL: intraepithelial lymphocytes; EMR: endoscopic mucosal resection.

${ }^{\mathrm{a}}$ Atypia was only noted focally and of mild in nature. 


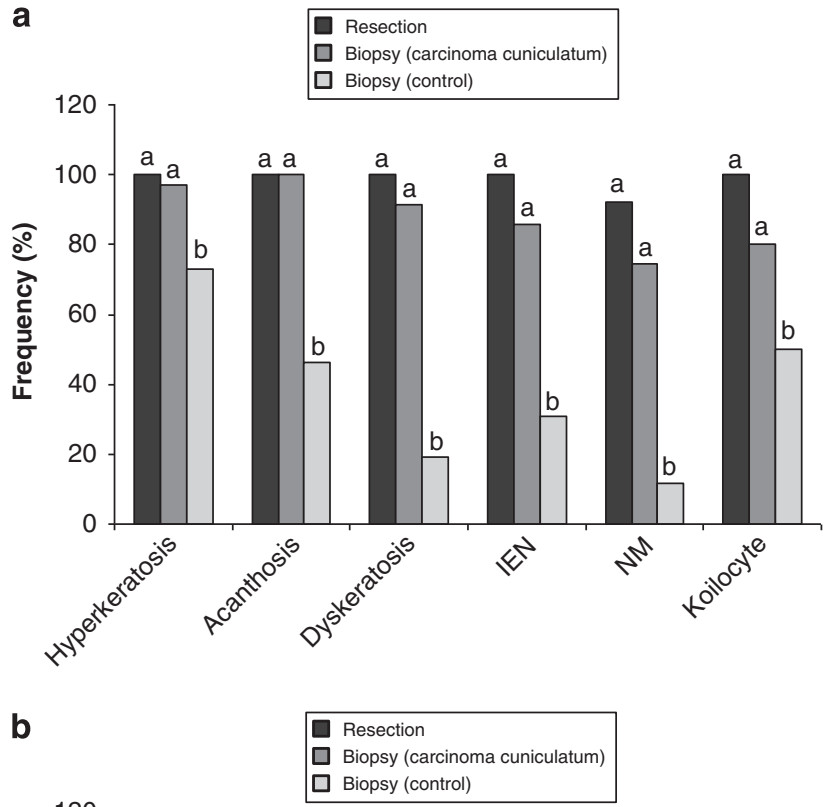

procedures (16/25) in 91\% patients (10/11) would be diagnostic, in comparison to the initial diagnostic rates of carcinoma of $9 \%$ (at biopsy level, $P<0.0001$ ), $12 \%$ (at procedure level, $P<0.0001$ ), and $27 \%$ (at patient level, $P<0.0001$ ). None of the 92 benign biopsies showed a score of $\geq 7(P<0.05)$.

\section{Discussion}

Carcinoma cuniculatum (epithelioma cuniculatum) was described by Ayrd et $a l^{1}$ in 1954 as a peculiar histologic variant of well-differentiated squamous cell carcinoma involving the foot. Bona fide examples of this tumor in the esophagus are exceedingly rare in the literature. To the best of our knowledge, only 11 cases involving the esophagus have been reported, which includes our previous series of 9 cases. ${ }^{6}$ A common histologic pattern characterized by hyperkeratosis, acanthosis, dyskeratosis, deep keratinization, keratin-filled cyst/burrows, koilocyte-like cells, intraepithelial neutrophils, intraepithelial neutrophilic microabscesses, and focal cytologic atypia was observed in our previous series based upon esophagectomy specimens. ${ }^{6}$ Due to the well-differentiated nature of this entity, definite diagnosis on endoscopic mucosal biopsy is extremely difficult. Our current study retrospectively reviewed the above histologic features in resection specimens and endoscopic mucosal biopsies prior to the definite diagnosis of carcinoma cuniculatum in a series of 11 patients. In addition, a semiquantitative histologic scoring scale was developed by summing all the above histologic features and assessed in the resected tumors of esophageal carcinoma cuniculatum, their corresponding endoscopic mucosal biopsies prior to resection, and a control group of endoscopic esophageal mucosal biopsies carrying benign diagnoses.

The frequency of hyperkeratosis, acanthosis, dyskeratosis, intraepithelial neutrophils, neutrophilic microabscesses, koilocyte-like cells, and keratin cyst/burrow in endoscopic esophageal biopsies taken from patients prior to their definite diagnosis of carcinoma cuniculatum was comparable to those seen in resected specimens. Other features such as deep keratinization, focal cytologic atypia, keratin cyst/burrows were seen in 63,51 , and $23 \%$ endoscopic mucosal biopsies, significantly lower than in resection specimens. Our results suggest that deep keratinization, atypia, and keratin cyst/burrows are either focal and/or located in the deep portion of the lesion and are less likely to be present in the endoscopic mucosal biopsy. Our results are in line with a previous study, which showed that nonpapillary squamous high-grade dysplasia was only present in the deep portion of the tumor but not the superficial endoscopic mucosal biopsy from verrucous carcinoma of the esophagus, a closely related variant of well-differentiated squamous cell carcinoma. ${ }^{8}$ Conventional diagnostic criteria of 
a
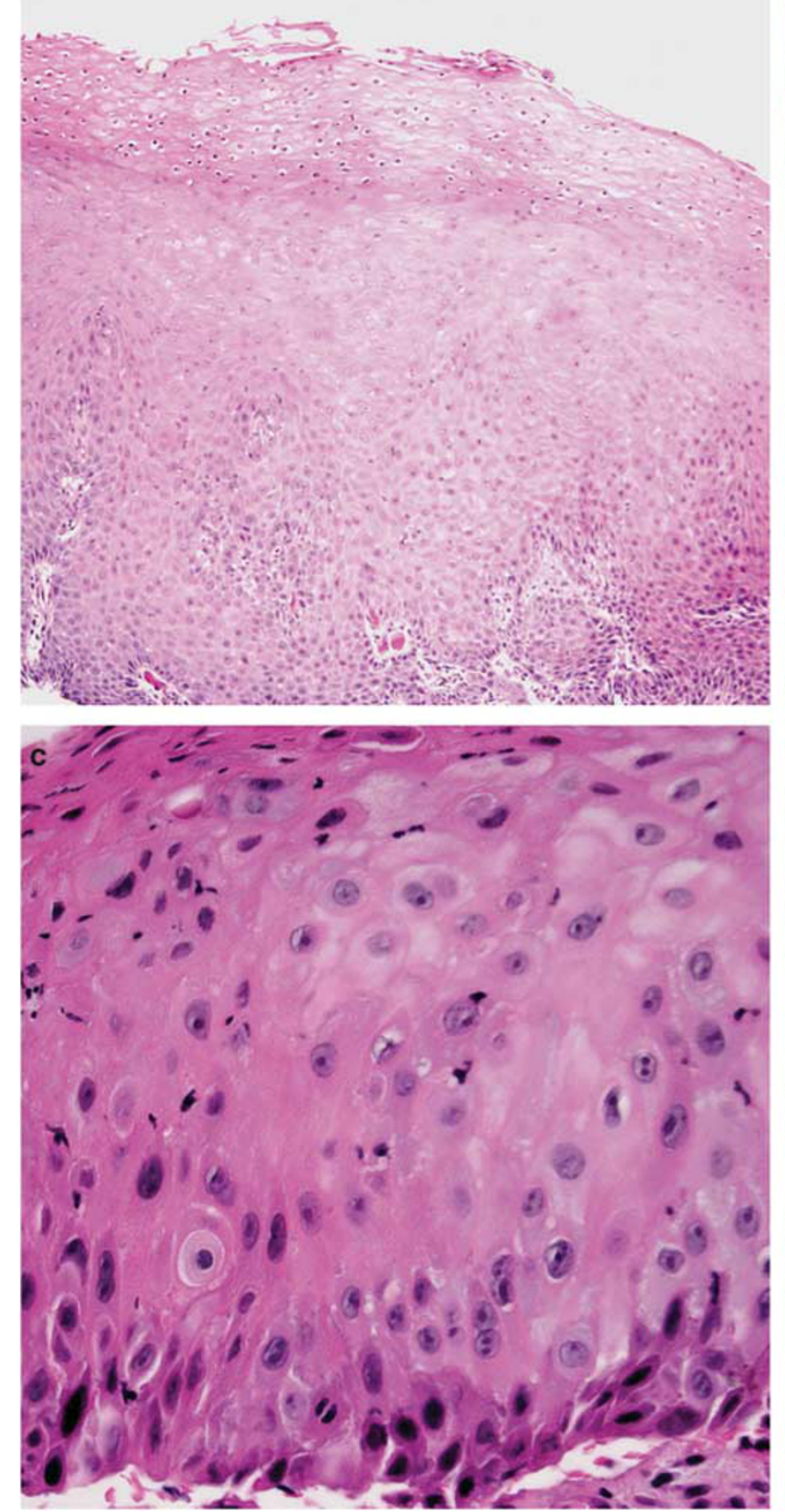
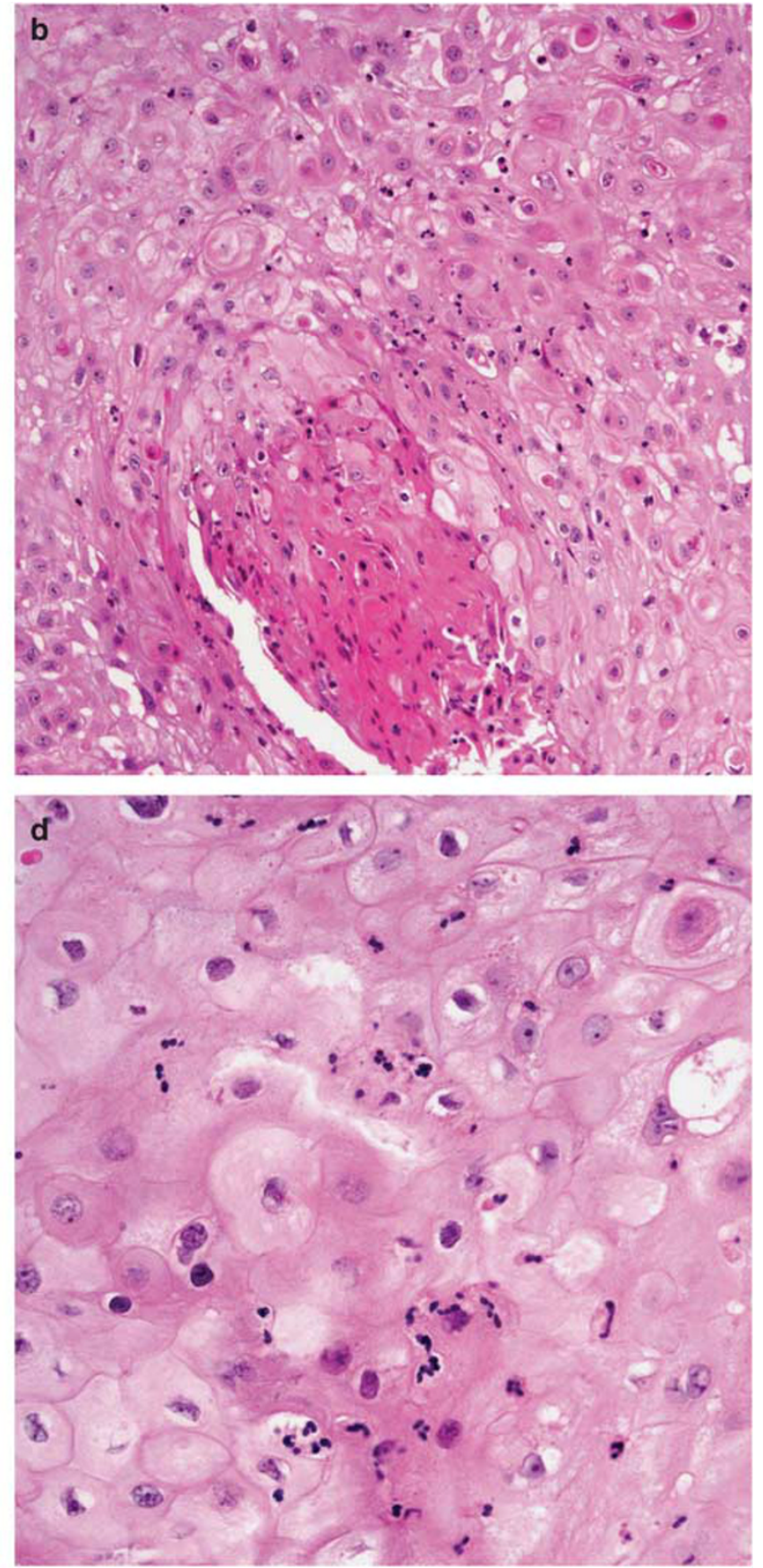

Figure 2 Histologic features of carcinoma cuniculatum on endoscopic mucosal biopsies. (a) Hyperkeratosis (hematoxylin and eosin stain, $100 \times$ ). (b) Dyskeratosis, deep keratinization, and intraepithelial neutrophilic infiltration (hematoxylin and eosin stain, $200 \times$ ). (c) Focal cytologic atypia (hematoxylin and eosin stain, $400 \times$ ). (d) Intraepithelial neutrophilic infiltration and koilocyte-like cells (hematoxylin and eosin stain, $400 \times$ ).

esophageal squamous cell carcinoma used by pathologists for endoscopic mucosal biopsy are heavily based on the presence of deep keratinization and atypia, particularly marked nuclear atypia, which may explain the low sensitivity of $9 \%$ observed in our study prior to our semiquantitative histologic assessment.

In this study, the original endoscopic biopsies were diagnosed as esophagitis or non-specific inflammation on 17 occasions including eight diagnoses of fungal esophagitis or changes suggestive of fungal esophagitis. The high frequency of Candida infection in our series supports the previous observation that Candida esophagitis is associated with carcinoma of the esophagus. ${ }^{9}$ Intraepithelial neutrophilic infiltration and intraepithelial neutrophilic microabscess are consistent findings in our current study, in both the resected and 


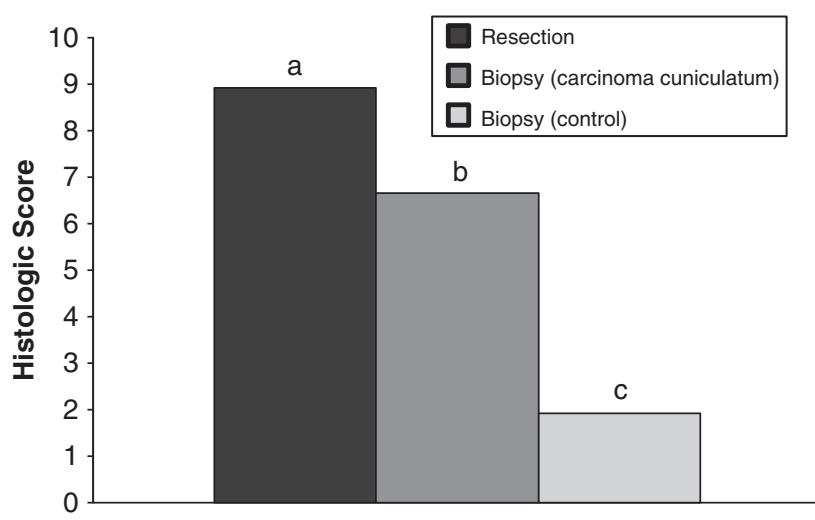

Figure 3 Comparison of histologic scores for carcinoma cuniculatum in resected specimens, endoscopic esophageal mucosal biopsy from patients with resection-proven carcinoma cinuculatum, and esophageal biopsies with benign diagnoses. All specimens were assessed for the presence of hyperkeratosis, acanthosis, dyskeratosis, deep keratinization, intraepithelial neutrophils, intraepithelial neutrophilic microabscess, focal cytologic atypia, koilocyte-like cells, and keratin-filled cyst/ burrows. Each feature, if present, was given 1 point, and the final histologic score was calculated for each biopsy by summing up the points. The mean scores for carcinoma cuniculatum in the resected specimens, endoscopic mucosal biopsies, and control esophageal biopsies were 8.91 (s.d. 0.30), 6.66 (s.d. 1.88), and 1.93 (s.d. 1.75). Letters a, b, c indicate significant differences among groups $(P<0.05)$. Table 3 . Pathologic findings on endoscopic mucosal biopsies prior to esophagectomy or EMR in patients with esophageal carcinoma cuniculatum.

endoscopic mucosal biopsies with carcinoma cuniculatum. The presence of neutrophilic inflammation and Candida organisms in endoscopic mucosal biopsies taken from an obvious esophageal mass should prompt a search for evidence of a well-differentiated squamous cell carcinoma such as carcinoma cuniculatum or verrucous carcinoma, as supported by our current series and the report by Tonna et al. ${ }^{8}$

The neutrophilic inflammation in the esophageal carcinoma cuniculatum may be due to superimposed Candida infection, bacterial overgrowth, local irritation/trauma secondary to obstruction, or an inflammatory response associated with the neoplastic process per se as it is also present in cases of carcinoma cuniculatum without Candida infection or bacterial overgrowth. While the exact mechanism of this intraepithelial neutrophilic infiltration remains unclear, a few previous studies appear to support that overexpression of transforming growth factor- $\beta 1$ (TGF $\beta 1$ ) in squamous cell neoplasm/ carcinoma may lead to neutrophilic inflammation via an interleukin 17 (IL-17)-dependent signaling pathway. ${ }^{10,11}$

Two other features consistently observed in the resected carcinoma cuniculatum are dyskeratosis and deep keratinization. Dyskeratosis is an indication of apoptosis, which has been shown to be an indicator of disturbed proliferation and growth. Deep keratinization indicates abnormal maturation and has been used as a criterion to diagnose invasive conventional squamous cell carcinoma.
While the mean histologic score in the endoscopic biopsies from patients with carcinoma cuniculatum was 6.66 (s.d. 1.88), significantly lower than that from the resected specimens, it was significantly higher than our control group. This finding suggests the potential utility of this histologic score in diagnosing carcinoma cuniculatum in endoscopic mucosal biopsies taken from an esophageal mass. Before applying the score sum described in this study, a definite diagnosis was only achieved in 3 of 11 cases $(27 \%)$ prior to esophagectomy $(n=9)$ or endoscopic mucosal resection $(n=2)$ with a diagnostic sensitivity $9 \%$ (3 of 35) at the biopsy level. Because suspicion for an atypical squamous proliferation was raised in the pathology reports of an additional seven biopsies from four patients (including one patient who had a diagnostic biopsy in a separately submitted biopsy taken during the same procedure), the maximal diagnostic sensitivity at the biopsy level was $26 \%$ and at the patient level was $54 \%$. Using a cutoff histologic score of 7 for carcinoma cuniculatum, $57 \%$ of biopsies (20/35) from $64 \%$ esophagogastroduodenoscopic procedures $(16 / 25)$ in $91 \%$ patients $(10 / 11)$ would be diagnostic, in comparison to the initial diagnostic rates of carcinoma of $9 \%$ (at biopsy level, $P<0.0001$ ), $12 \%$ (at procedure level, $P<0.0001$ ), and $27 \%$ (at patient level, $P<0.0001$ ). Moreover, none of the 92 benign biopsies showed a score of $\geq 7$ $(P<0.05)$. Our study showed that a semiquantitative histologic evaluation of esophageal mucosal biopsies improves the diagnostic sensitivity from 9 to $57 \%$ in a single biopsy specimen from patients with carcinoma cuniculatum, with $100 \%$ specificity.

Before applying the score sum described in this study, the mean duration between initial endoscopic biopsy performed at our institution to the resectionproven diagnosis was 16 months (range: 0.25-121 months, median: 1 month). The patient with the longest interval between the initial endoscopic biopsy to the resection-proven diagnosis (121 months (case 10)) died from tracheal recurrence at 8 months after the diagnosis, suggesting that early diagnosis of this entity is essential.

Our control group consisted of a variety of consecutively diagnosed benign histologic diagnoses. Because intraepithelial neutrophils and neutrophilic microabscesses may be seen in bullous diseases, some of which have been reported to be associated with the development of squamous cell carcinoma of the esophagus, we included nine biopsies from five patients with bullous diseases (not further characterized) in our control group. In the control group, eight biopsies had a score of 5 $(n=4)$ or $6(n=4)$. The biopsies with a score of 5 were from patients with Candida esophagitis with renal transplant $(n=1)$ or with a subepithelial nodule $(n=1)$, bullous disease with stricture $(n=1)$, and erosive reflux esophagitis $(n=1)$. Follow-up endoscopic mucosal biopsy showed improved histologic score (from 5 to 4 ) in two cases. 
The biopsies with a score of 6 were erosive esophagitis (from a patient with end-stage renal disease), active esophagitis (from patients with stricture at the esophagogastric junction $(n=1)$ or of the entire esophagus $(n=1)$ ), and Candida esophagitis from a patient with radiation-induced stricture of the cervical esophagus for the treatment of laryngeal squamous cell carcinoma. Follow-up endoscopic mucosal biopsy showed improved histologic score to 0 in the patient who had erosive esophagitis (from 6 to 0 ) and in the patient with active esophagitis with stricture at the EGJ (from 6 to 3 ). The remaining four cases with a score of 5 or 6 were lost to follow-up. Our results suggest that patients with a histologic score of 5 and 6 should be carefully followed-up clinically as $4(50 \%)$ had esophageal stricture, 2 (25\%) Candida esophagitis, 1 erosive reflux disease (13\%), and $1(13 \%)$ erosive esophagitis in end-stage renal disease, all conditions that are associated with the development of squamous cell carcinoma.

There are overlapping histologic features between carcinoma cuniculatum and verrucous carcinoma. Verrucous carcinoma of the esophagus more often presents with $\mathrm{T} 4$ disease and carries a high mortality. ${ }^{6,12}$ It is not clear whether the difference in outcome is due to earlier detection and treatment of the same disease or whether true biologic differences exist between these entities. It may be impossible to differentiate these entities on endoscopic mucosal biopsies as both entities pose enormous diagnostic difficulties as shown in our current studies as well as in the previous reports..$^{8,13}$

As esophageal squamous mucosa can undergo marked reactive changes, we caution that a diagnosis of carcinoma cuniculatum should not be solely based on the histologic assessment, even with the semiquantitative approach described here. Rather, the diagnosis should only be made with a strict clinicopathologic correlation. In fact, it is our opinion that the process of 'scoring' should not be initiated if there is no strong clinical suspicion of this tumor.

In summary, esophageal carcinoma cuniculatum is a rare variant of well-differentiated squamous cell carcinoma with a characteristic constellation of morphologic features; many of which are also present on the endoscopic mucosal biopsy. A semiquantitative histologic scoring system is proposed; using a cutoff score of 7 for endoscopic mucosal biopsies from esophageal mass greatly improves the diagnostic sensitivity to $57 \%$ with $100 \%$ specificity. Diagnosis of this entity at an early stage may significantly improve the clinical outcome. Larger studies through multi-center collaboration are needed to confirm our current findings.

\section{Disclosure/conflict of interest}

The authors declare no conflict of interest.

\section{References}

1 Ayrd I, Johnson HD, Lennox B, et al. Epithelioma cuniculatum: a variety of squamous carcinoma peculiar to the foot. Br J Surg 1954;42:245-250.

2 McKee PH, Wilkinson KD, Black MM, et al. Carcinoma (epithelioma) cuniculatum: a clinico-pathological study of nineteen cases and review of the literature. Histopathology 1981;5:425-436.

3 Barreto JE, Velasquez EF, Ayala E, et al. Carcinoma cuniculatum: a distinct variant of penile squamous cell carcinoma. Am J Surg Pathol 2007;31:71-75.

4 Puxeddu R, Cocco D, Parodo G, et al. Carcinoma cuniculatum of the larynx: a rare clinicopathological entity. J Laryngol Otol 2008;122:1118-1123.

5 Gassler N, Helmke B, Schweigert HG, et al. (Carcinoma cuniculatum of the oral cavity. A contribution to the differential diagnosis of potentially malignant papillary lesions of mouth mucosa). Pathologe 2002;23: 313-317.

6 Landau M, Goldblum JR, DeRoche T, et al. Esophageal carcinoma cuniculatum: report of 9 cases. Am J Surg Pathol 2012;36:8-17.

7 De Petris G, Lewin M, Shoji T. Carcinoma cuniculatum of the esophagus. Ann Diagn Pathol 2005;9:134-138.

8 Tonna J, Palefsky JM, Rabban J, et al. Esophageal verrucous carcinoma arising from hyperkeratosis plaques associated with human papilloma viral type 51 . Dis Esophagus 2010;23:E17-E20.

9 Bhatia V, Kochhar R, Talwar P, et al. Association of candida with carcinoma of esophagus. Indian J Gastroenterol 1989;8:171-172.

10 Natsugoe S, Xiangming C, Matsumoto M, et al. Smad4 and transforming growth factor $\beta 1$ expression in patients with squamous cell carcinoma of the esophagus. Clin Cancer Res 2002;8:1838-1842.

11 Mohammed J, Ryscavage A, Perez-Lorenzo R, et al. TGF $\beta 1$-induced inflammation in premalignant epidermal squamous lesions requires IL-17. J Invest Dermatol 2010;130:2295-2303.

12 Osborn NK, Keate RF, Trastek VF, et al. Verrucous carcinoma of the esophagus: clinicopathophysiologic features and treatment of a rare entity. Dig Dis Sci 2003;48:465-474.

13 Devlin S, Falck V, Urbanski SJ, et al. Verrucous carcinoma of the esophagus eluding multiple sets of endoscopic biopsies and endoscopic ultrasound: a case report and review of the literature. Can J Gastroenterol 2004;18:459-462. 